\section{JTI}

JOURNAL OF

TRAUMA AND INJURY

\title{
Delayed Surgical Management of Traumatic Pseudoaneurysm of the Ascending Aorta in Multiple Trauma
}

\author{
Dae Sung Ma, M.D., Sung Jin Kim, M.D., Seok Joo, M.D., Sung Youl Hyun, M.D., \\ Yang Bin Jeon, M.D.
}

Department of Trauma Surgery, Trauma Center, Gachon University Gil Medical Center, Incheon, Korea

Received: December 14, 2017

Revised: January 2, 2018

Accepted: February 5, 2018

Correspondence to

Yang Bin Jeon, M.D.

Department of Trauma Surgery, Trauma Center, Gachon University Gil Medical Center, 21 Namdong-daero 774beon-gil, Namdong-gu, Incheon 21565, Korea

Tel: $+82-32-460-8316$

Fax: +82-32-460-2372

E-mail: junyb@gilhospital.com
Traumatic blunt aortic injury especially on proximal ascending aorta is a rare injury with a few reports. Generally emergency surgical management was performed. In this case, however, in multiple trauma with brain injury, emergency surgical management of aortic injury might result in unexpected secondary injury of the brain. Herein, we report a case of a 33-year-old man who was driving a truck was injured in a head-on collision. Evaluation revealed a pseudoaneurysm on his ascending aorta concomitant with epidural hemorrhage. He was treated by surgical management of his ascending aorta after 3 days from accident. There were no postoperative and neurologic complications and the patient was discharged after 18 days.

Keywords: Trauma; Pseudoaneurysm; Ascending aorta

\section{INTRODUCTION}

Blunt traumatic aortic injury, expecially non-isthmus aortic injuries is known lethal, and most of patients are died at scene, or within the first 24 hours in hospital [1,2]. In general, proximal ascending aorta injury is treated with emergency surgical repair. However, multiply traumatized patient combined with proximal ascending aorta injury and brain injury require careful approach to manage, because an emergency surgical repair of ascending aorta is possible to result in secondary injury of CNS. We report the case of a successful surgical repair of proximal ascending aortic injury with brain injury after 3 days from trauma. (http://creativecommons.org/licenses/by-nc/4.0/) which permits unrestricted noncommercial use, distribution, and reproduction in any medium, provided the original work is properly cited. 


\section{CASE REPORT}

A 33-year-old man who was driving a truck was injured

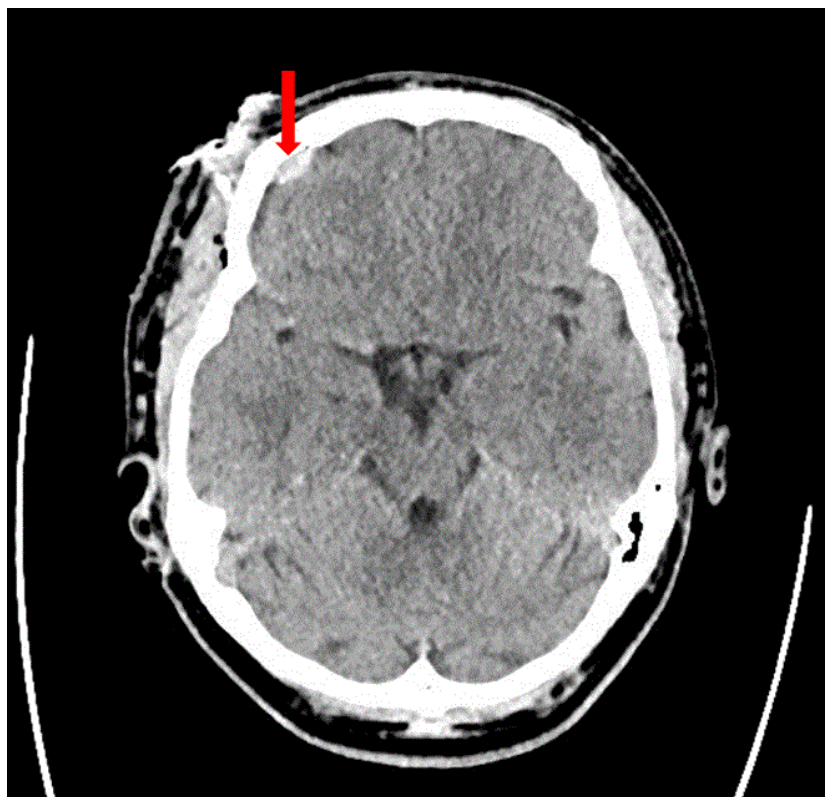

Fig. 1. Computed tomography of brain, Traumatic epidural hemorrhage (arrow). in a head-on collision. He visited our trauma center complaining of facial and thoracic pain. Physical examination revealed lacerations on the forehead and right lower lip of about $6 \mathrm{~cm}$ and $5 \mathrm{~cm}$, respectively, with bone exposure. Initial vital signs were: blood pressure 145/87 mmHg, heart rate 86 beats per minute, and respiratory rate 21 breaths per minute. The patient was alert but slightly confused. Radiology workup included computed tomography (CT) of the brain, thorax, abdomen, and pelvis. The brain CT revealed slight localized epidural hematoma in the right frontal lobe area, slight pneumocephalus, and fracture in the right frontal bone (Fig. 1). The thoracic CT revealed a small pseudoaneurysm on the left side of the ascending aorta with an intramural hematoma on the ascending aorta and aortic arch, slight hemopericardium, and a longitudinal fracture in the manubrium with retrosternal hematoma. There was also bilateral pulmonary contusion (Fig. 2). Three hours after arriving to the hospital, a repeat brain CT revealed a slight increase in the localized epidural hematoma in the right frontal lobe, compared to the last examination. The patient was nearly alert and his vital signs were stable: blood pressure 120/74 $\mathrm{mmHg}$, heart rate 81 beats per minute, and oxygen satu-
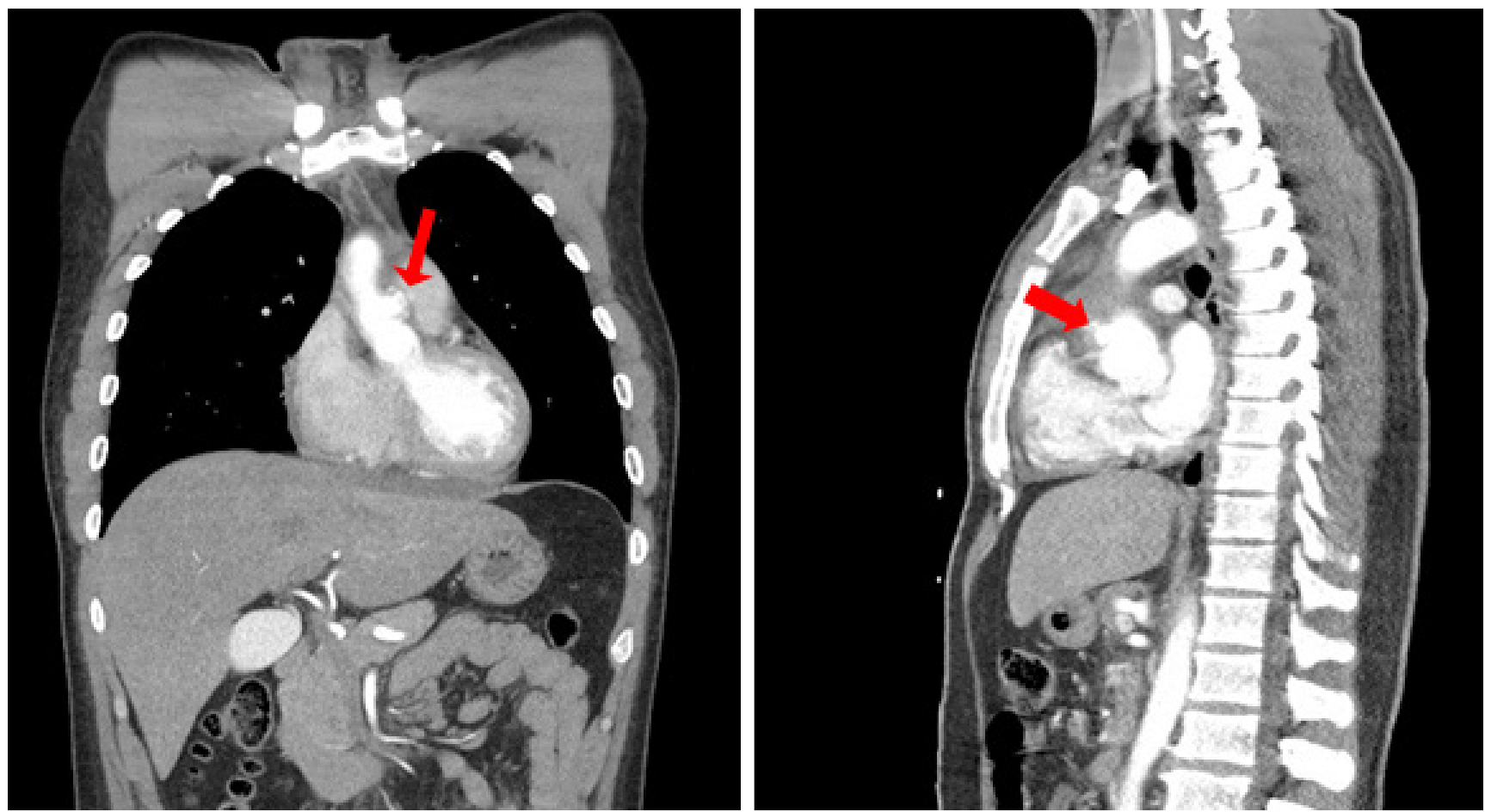

Fig. 2. Computed tomography of thorax, pseudoaneurysm of ascending aorta (arrow) 

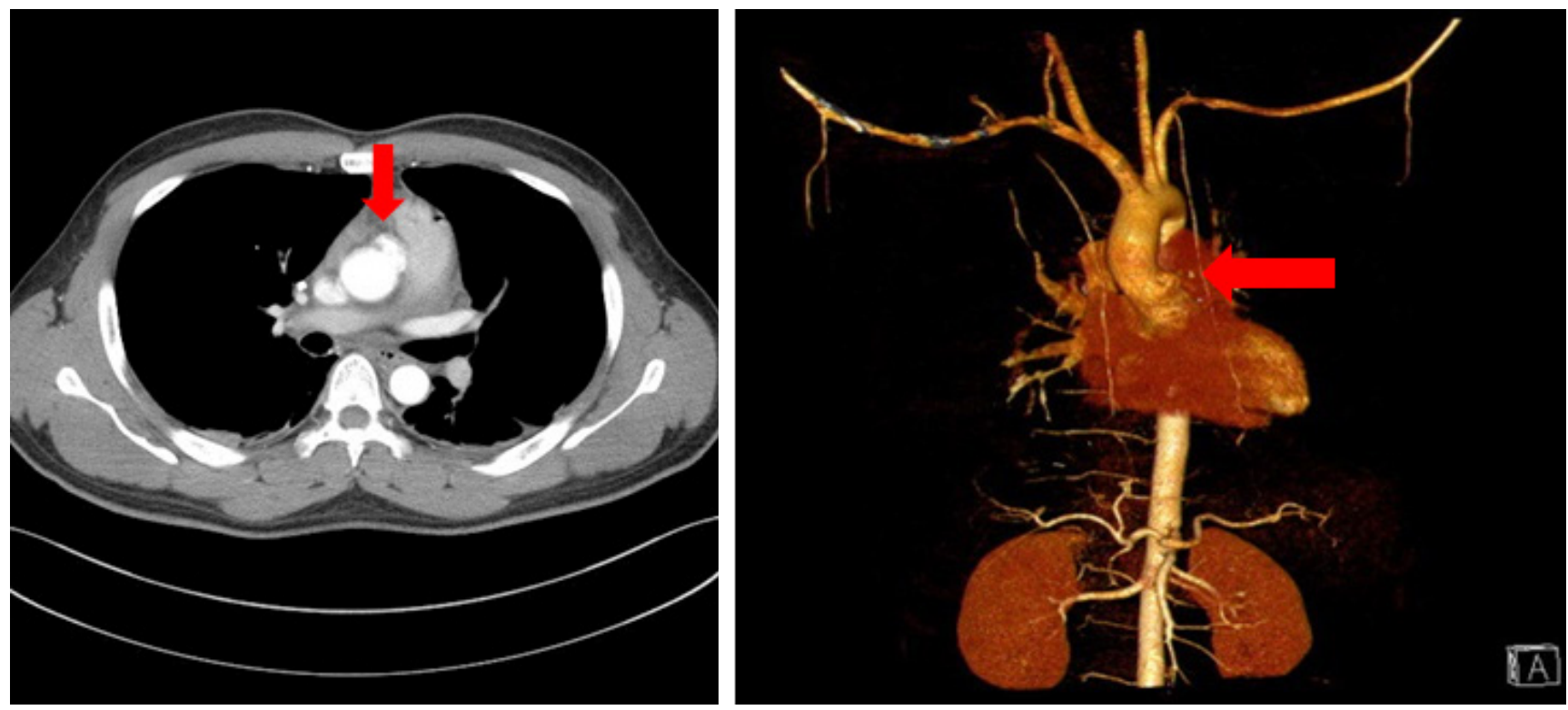

Fig. 3. Computed tomography of thoracic aortography after 1 day. Pseudoaneurysm of ascending aorta (arrow).
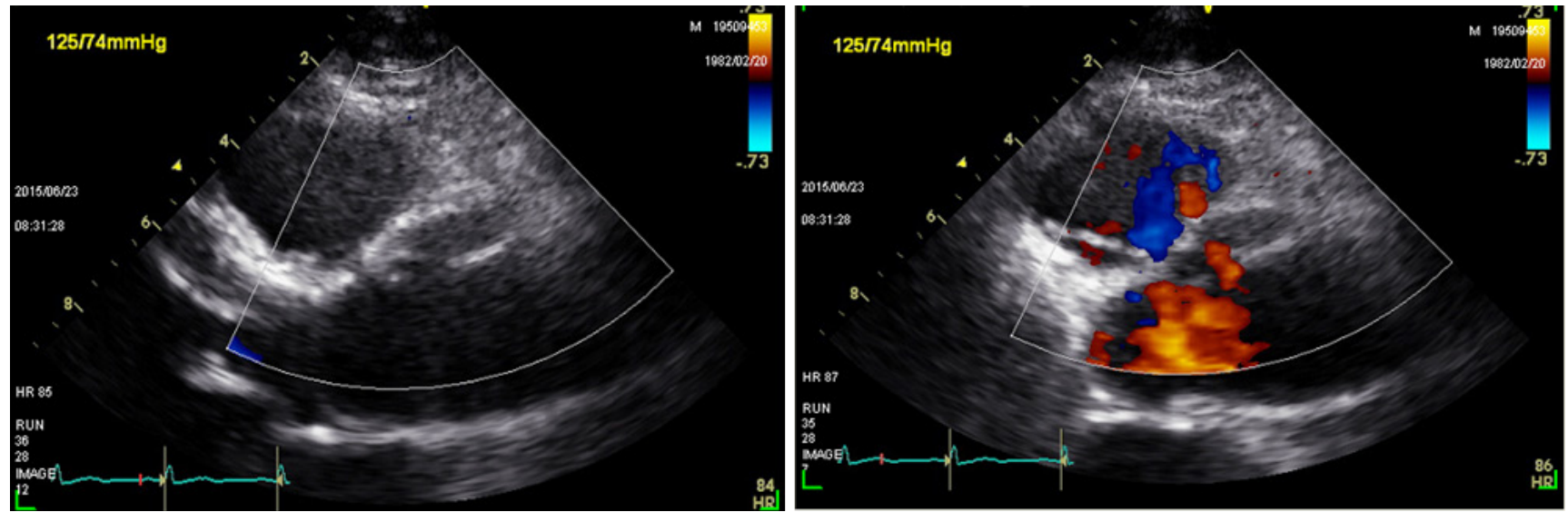

Fig. 4. Preoperative transesophageal echocardiography of ascending aorta pseudoaneurysm.

ration $98 \%$, with $\mathrm{O}_{2}$ at $3 \mathrm{~L} / \mathrm{min}$ via a nasal cannula. Primary suturing was performed for the open wound on his forehead and lower lip and the patient was transferred to the Trauma Intensive Care Unit (TICU) for close monitoring.

Repeat thoracic aorta CT was performed for a more detailed evaluation and another prominent pseudoaneurysm was found on the left side of the ascending aorta (Fig. 3). Two days later, brain CT was performed to ascertain the feasibility of thoracic surgery and to evaluate the previous lesion. However, there was no change in the epidural hematoma in the right frontal lobe. Surgical treatment of the ascending aorta pseudoaneurysm was performed three days after the trauma, and a false aneurysmal sac was noted between the right coronary artery and pulmonary artery along the left ascending aorta. The aneurysm resection was performed after incision in side of false aneurysm adventitia. The intima tearing was observed directly upper portion from L-R commisure, upper margin of right sinus of Valsava. The progressive dissection was observed in a surgical field. Thus, ascending aorta reconstruction with graft (hemashield $22 \mathrm{~mm}$ ) and commissure resuspension of the left-to-right commissural were performed. Further, transesophageal echocardiography (TEE) 

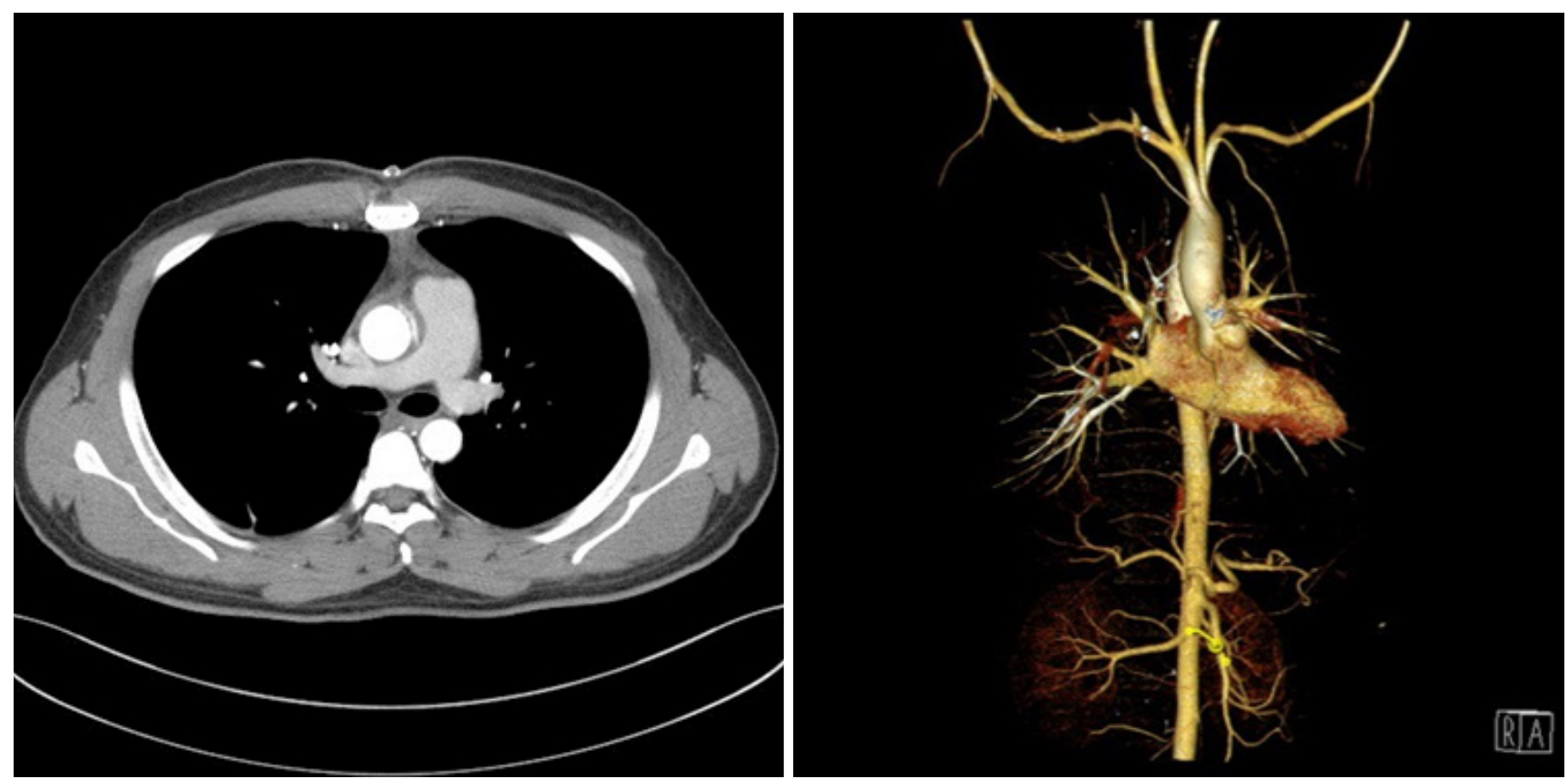

Fig. 5. Postoperative thoracic computed tomography aortography after 7 days.

performed in the operating room revealed a traumatic aortic dissection above the sinotubular junction and a small amount of pericardial effusion before the cardiopulmonary bypass (Fig. 4). According to the Vancouver classification, our case was classified to Grade III blunt aorta injury. Postoperative TEE revealed mild depressed left ventricular systolic function and there was no abnormal flow in the aortic dissection and aortoplasty site. Postoperative thoracic aorta $\mathrm{CT}$ after 7 days revealed no definite hematoma in the ascending aorta and aorta arch (Fig. 5). The patient was transferred to the Department of Plastic Surgery after 9 days and underwent open reduction and internal fixation for the tripod fracture associated with orbital floor fracture. On the 18th hospital day, he was discharged without surgical complications.

\section{DISCUSSION}

Blunt traumatic aorta injury is a leading cause of death after blunt trauma. Most patients with aortic injury are reported to be died before transfer to hospital, and up to $50 \%$ of those alive on arrival die within the first 24 hours [1]. Blunt aortic injury is rare and fatal. In an autopsy study on blunt aorta injury in 241 patients, the majority of aortic lacerations occurred at the classic isthmus site (58\%) and the ascending aorta (8\%) [2]. However, a surgical study demonstrated $92 \%$ of aortic lacerations at the isthmus and 3\% in the ascending aorta [3], suggesting that non-isthmus aortic injuries may be more lethal [2].

The mechanism of injury including rapid deceleration, shearing forces, osseous pinch, and hydrostatic forces account for the severity of blunt aortic injury. However, among these, no definitive theory has been established. Depending on the aortic injury site, the mechanism can be described as a single force or combination of forces. In our case, sternal fracture, intramural hematoma of the ascending aorta and aortic arch, osseous pinch and hydrostatic forces may be the mechanism of injury.

Since the first report of the endovascular repair of traumatic aortic injury in 1997 [4], it has been accepted as a method for reducing mortality, with fewer blood transfusions and lower risk of paraplegia compared with traditional surgical repair [5]. Endovascular aortic repair for proximal ascending aorta injury has been performed occasionally in poor condition of surgical management, but surgical treatment is still a choise of treatment [6]. In our knowledge, there was no report about TEVAR for 
ascending aorta injury in domestic, and it was not a treatment option in our case. And, in multiple traumas, early intervention (within 24 hours) for blunt traumatic aorta injury with traumatic brain injury is reported to worsen neurologic deterioration [7]. Thus, the urgent surgical repair of traumatic aortic injury was performed after critical management and evaluation for associated injury including traumatic brain injury.

In blunt aortic injury (BAI) higher than grade II of Vancouver classification, our treatment policy is TEVAR unique to descending thoracic aorta if it was not impending to rupture. However, blunt aortic injury on ascending aorta is very rare and there is no established protocol. Based on surgical management in Standford type A dissection, if it is not contraindication, emergency surgical repair may be a useful treatment for ascending aorta injury.

The proximal ascending aorta pseudoaneurysm after blunt trauma is an unusual presentation of BAI. We demonstrate the successful surgical management of traumatic proximal ascending aorta injury combined with traumatic brain injury.

\section{REFERENCES}

1. Jamieson WR, Janusz MT, Gudas VM, Burr LH, Fradet
GJ, Henderson C. Traumatic rupture of the thoracic aorta: third decade of experience. Am J Surg 2002;183:571-5.

2. Burkhart HM, Gomez GA, Jacobson LE, Pless JE, Broadie TA. Fatal blunt aortic injuries: a review of 242 autopsy cases. J Trauma 2001;50:113-5.

3. Symbas PN, Tyras DH, Ware RE, Diorio DA. Traumatic rupture of the aorta. Ann Surg 1973;178:6-12.

4. Kato N, Dake MD, Miller DC, Semba CP, Mitchell RS, Razavi MK, et al. Traumatic thoracic aortic aneurysm: treatment with endovascular stent-grafts. Radiology 1997;205:657-62.

5. Demetriades D, Velmahos GC, Scalea TM, Jurkovich GJ, Karmy-Jones R, Teixeira PG, et al. Operative repair or endovascular stent graft in blunt traumatic thoracic aortic injuries: results of an American Association for the Surgery of Trauma Multicenter Study. J Trauma 2008;64:561-70; discussion 570-1.

6. Nienaber CA, Sakalihasan N, Clough RE, Aboukoura M, Mancuso E, Yeh JS, et al. Thoracic endovascular aortic repair (TEVAR) in proximal (type A) aortic dissection: Ready for a broader application? J Thorac Cardiovasc Surg 2017;153:S3-11.

7. Rabin J, Harris DG, Crews GA, Ho M, Taylor BS, Sarkar $\mathrm{R}$, et al. Early aortic repair worsens concurrent traumatic brain injury. Ann Thorac Surg 2014;98:46-51; discussion $51-2$. 